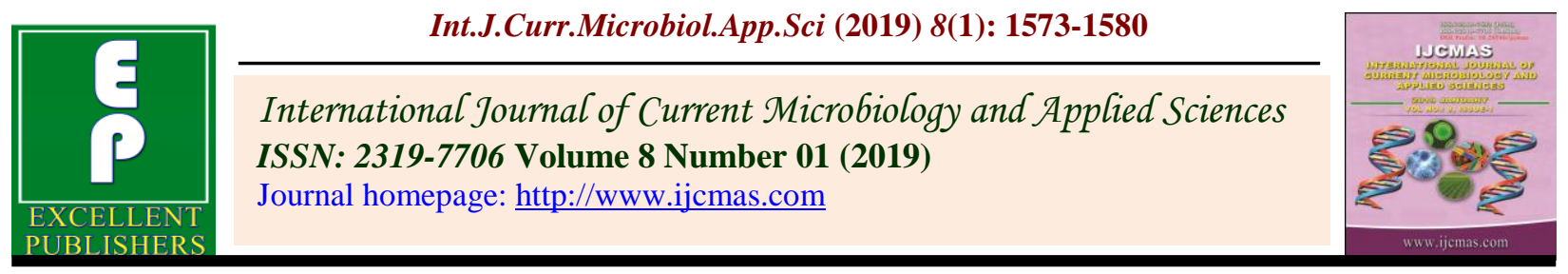

Original Research Article

https://doi.org/10.20546/ijcmas.2019.801.165

\title{
New Approaches of Management for Apple Blossom Thrip (ABT) in Apple Orchards of Kashmir Valley
}

\author{
Muneer Ahmad*, M. Ayoub Manto, Sajad Mohu Din and S.S. Pathania \\ Division of Entomology, Sher-e-Kashmir University of Agricultural Sciences and Technology, \\ Shalimar Srinagar, Kashmir, J\&K 190025, India \\ *Corresponding author
}

\begin{abstract}
A B S T R A C T
Keywords

Bio-efficacy,

Orchards,

Thiocloroprid,

Apple blossom

thrips

Article Info

Accepted:

12 December 2018

Available Online:

10 January 2019

Apple blossom thrips (Thysanoptera: Thripidae) is serious pest of apple bloom in some areas of Bandipora and Anantnag of kashmir valley. A field trials were laid out at Dangerpora Kullar, Luckbown (District Anantnag) and Arin and bagh (District Bandipora) in a completely Randomized Block Design during 2015 - 2017. Apple trees of variety "Red Delicious", 13-16 years of age were selected for the field trial. The highest pest incidence (5-8) thrips per flower was recorded in Madar bandipora. The spraying of the treatments, viz., Thiochloroprid @ 0.3, 0.4 and $0.5 \mathrm{ml} / \mathrm{L}$ of water, chlorpyriphos 20 EC @ $0.02 \%$, dimethoate 30 EC @ $0.05 \%$ and water as control. The overall bio efficacy of the insecticides evaluated against apple blossom thrip (ABT) was in the order of Code 188 $(0.5 \mathrm{ml} / \mathrm{L})>$ chlorpyriphos $20 \mathrm{EC}(0.02 \%)>$ dimethoate $30 \mathrm{EC}(0.05 \%)>$ Code thiocloroprid $(0.4 \mathrm{ml} / \mathrm{L})>$ thiocloroprid $(0.3 \mathrm{ml} / \mathrm{L})$. While comparing the bioefficacy of the treatments on fruit yield (A Grade-fruit boxes / tree), the order of efficacy was thiocloroprid $(0.5 \mathrm{ml} / \mathrm{L})>$ dimethoate $30 \mathrm{EC}(0.05 \%)>$ thiocloroprid $(0.4 \mathrm{ml} / \mathrm{L})>$ chlorpyriphos $20 \mathrm{EC}(0.02 \%)>$ thiocloroprid $(0.3 \mathrm{ml} / \mathrm{L})$.

\section{Introduction}

Apple blossom Thrip (Thysanoptera: Thripidae) damages the fruit of a number of crops. Apple blossom thrip is one of the alarming pests of apple (Malus domestica Borkh) in Kashmir. Feeding by young nymphs and adults produces distinct symptoms on flower petals as they have rasping type of mouth parts. The female parts of the flower are affected by the pest with the result fruit setting is reduced drastically. The traditional method of sampling apple blossoms to check for numbers of thrips is to

examine samples of buds, pulling them apart and shaking them onto a white paper or Vaseline - smeared Petri-dish. Many factors have to be taken into consideration when determining an economic threshold for thrips. Chemical control is one of the most effective and practical method available to the orchardists for the effective control of apple blossom thrip (ABT). For the last few years, the pest has been appearing in alarming form in most apple growing areas, suck sap from the flowers resulting in reduced fruit set and huge economic losses to apple. The bioefficacy of various insecticides against the
\end{abstract}


pest, were evaluated in district Anantnag and Bandipora Kashmir for the first time and the results are presented here.

\section{Materials and Methods}

A field trials were laid out at Dangerpora Kullar and Luckbown (District Anantnag) and Arin and bagh bandipora (District bandipora) in a completely Randomized Block Design during 2015 - 2017, respectively. Apple trees of variety "Red Delicious", 13-16 years of age were selected for the field trial. The survey was conducted in hot pockets of two districts where apple orchards are infested.The spraying of the treatments, viz., Thiocloroprid @ 0.3, 0.4 and $0.5 \mathrm{ml} / \mathrm{L}$ of water, chlorpyriphos 20 EC @ 0.02\%, dimethoate 30 EC @ $0.05 \%$ and water as control, each replicated four times with a single tree as a replicate, was done with the help of a power sprayer at pink bud stage and the observations on the number of apple blossom thrips (ABT) per cluster of flowers before and after the treatment applications were counted at subsequent intervals of $1^{\text {st }}$, $3^{\text {rd }}, 5^{\text {th }}, 7^{\text {th }}$ and $10^{\text {th }}$ day after treatment (DAT) by taking a random sample of four clusters from outer, middle and innermost parts of the tree canopy. Per cent mortality was worked out by computing the difference between pre and post treatment populations of the apple blossom thrip (ABT) by applying Abbot's (1925) formula. The observations on the fruit yield (fruit boxes / tree) were recorded after harvest of the apple crop from the experimental trees. The data was subject to analysis of variance and critical difference at $5 \%$ level of significance was worked out. The yield of A-Grade boxes per treatment was recorded at the time of harvest.

\section{Results and Discussion}

The incidence of Apple blossom thrips were recorded in different places which are hot pockets of this insect pest. The highest pest incidence was recorded in Madar which recorded 5-8 thrips /flower which is more than ETL level of the pest followed by Arin Bandipora which recorded 5-7 thrips/flower while as least 3-5 thrips/flower were recorded in Dangerpora ananthnag Kashmir (Table 1).

Bio-efficacy of Thiocloroprid against Apple blossom Thrip on apple at Arin (Bandipora) during 2015

Amongst the insecticides, Thiocloroprid resulted 51.11, 74.33, 83.33 and 93.66\%; 48.26, 74.37, 87.11, 87.11 and 94.95\%; 52.62, $76.46,84.49,89.74$ and $93.66 \%$ mortality of the apple blossom thrip population at $0.3,0.4$ and $0.5 \mathrm{ml} / \mathrm{L}$ of water in comparison to check $(3.09,3.09,6.09,6.09$ and $9.09 \%) 1^{\text {st }}, 3^{\text {rd }} 5^{\text {th }}$, $7^{\text {th }}$ and $10^{\text {th }}$ DAT, respectively. Similarly chlorpyriphos $20 \mathrm{EC}$ and dimethoate $30 \mathrm{EC}$ resulted 55.55, 73.88, 82.12, 90.87 and 46.83, $74.33,81.66,81.66$ and $97.91 \%$ mortality of apple blossom thrip population at 0.02 and $0.05 \%$ concentrations at $1^{\text {st }}, 3^{\text {rd }} 5^{\text {th }}, 7^{\text {th }}$ and $10^{\text {th }}$ DAT. Thiocloroprid @ $0.5 \mathrm{ml} /$ lit. of water resulted highest mean mortality $(80.19 \%)$ Whereas, least mean percent mortality $(71.7 \%)$ at $0.3 \mathrm{ml} /$ lit of water while as Thiocloroprid exhibited $78.38 \%$ mean mortality @ 0.4ml/litre of water. All the treatments were statistically different from control (Table 2).

\section{Bio-efficacy of against apple blossom thrip on apple at Arin (Bandipora during 2016)}

Amongst the insecticides, Thiocloroprid resulted 20.77, 63.00, 77.77, 81.55 and $92.66 \% ; 23.09,61.59,80.83,80.83$ and $92.37 \% ; 33.33,66.06,87.77,88.88$ and $95.66 \%$ mortality of the apple blossom thrip (ABT) population at $0.3,0.4$ and $0.5 \mathrm{ml} / \mathrm{L}$ of water in comparison to check $(6.00,7.00$, $8.00,10.33$ and $11.33 \%) 1^{\text {st }}, 3^{\text {rd }} 5^{\text {th }}, 7^{\text {th }}$ and $10^{\text {th }}$ DAT, respectively. Similarly 
chlorpyriphos $20 \mathrm{EC}$ and dimethoate $30 \mathrm{EC}$ resulted $43.71,71.85,81.23,87.52$ and $96.90 \%$; $35.69,67.84,78.56,89.28$ and $92.92 \%$ mortality of apple blossom thrip population at 0.02 and $0.05 \%$ concentrations at $1^{\text {st }}, 3^{\text {rd }} 5^{\text {th }}$, $7^{\text {th }}$ and $10^{\text {th }}$ DAT, respectively. Amongst thiocloroprid @ 0.5ml/ lit. of water resulted highest mean mortality (79.34\%) Whereas, least mean percent mortality $(67.15 \%)$ at $0.3 \mathrm{ml} /$ lit of water while as thiocloroprid exhibited $67.75 \quad \% \quad$ mean mortality @ $0.4 \mathrm{ml} /$ litre of water. All the treatments were statistically different from control (Table 3 ).

\section{Bio-efficacy of Thiocloroprid against Apple} blossom thrip on apple at Dangerpora Kullar (Anantnag) during 2015

Amongst the insecticides, Thiocloroprid resulted 76.88, 84.95, 90.66, 95.45 and $98.48 \% ; 86.89,91.46,94.48,99.26$ and 100\%; 93.08, 95.90, 99.36, 100 and 100\% mortality of the apple blossom thrip population at $0.3,0.4$ and $0.5 \mathrm{ml} / \mathrm{L}$ of water in comparison to check $(38.17,28.57,23.08$, 21.12 and $18.51 \%) 1^{\text {st }}, 3^{\text {rd }} 5^{\text {th }}, 7^{\text {th }}$ and $10^{\text {th }}$ DAT, respectively. Similarly chlorpyriphos $20 \mathrm{EC}$ and dimethoate $30 \mathrm{EC}$ resulted 88.68 , 94.12, 96.73, 99.54 and 100\%; 88.80, 94.20,
98.64, 100 and 100\% mortality of apple blossom thrip population at 0.02 and $0.05 \%$ concentrations in comparison to check (38.17, $28.57,23.08,21.12$ and $18.51 \%) 1^{\text {st }}, 3^{\text {rd }} 5^{\text {th }}$, $7^{\text {th }}$ and $10^{\text {th }}$ DAT, respectively (Table 4 and Fig. 1).

Bio-efficacy of Code 118 against apple blossom thrip on apple at Luckbown (Anantnag) during 2017

Amongst the insecticides, thiocloroprid resulted 79.16, 85.64, 91.14, 94.35 and $98.14 \% ; 86.08,90.62,96.26,98.73$ and $100 \%$; 91.17, 95.93, 98.90, 100.00 and $100 \%$ mortality of the apple blossom thrip population at $0.3,0.4$ and $0.5 \mathrm{ml} / \mathrm{L}$ of water in comparison to check $(29.30,25.28,21.99$, 19.19 and $15.72 \%) 1^{\text {st }}, 3^{\text {rd }} 5^{\text {th }}, 7^{\text {th }}$ and $10^{\text {th }}$ DAT, respectively. Similarly chlorpyriphos 20 EC and dimethoate $30 \mathrm{EC}$ resulted 89.63, 93.94, 98.27, 99.43 and $100 \%$; 86.65, 92.43, 96.93, 99.38 and $100 \%$ mortality of apple blossom thrip population at 0.02 and $0.05 \%$ concentrations in comparison to check (29.30, $25.28,21.99,19.19$ and $15.72 \%) 1^{\text {st }}, 3^{\text {rd }} 5^{\text {th }}$, $7^{\text {th }}$ and $10^{\text {th }}$ DAT, respectively (Table 5 and Fig. 2).

Table.1 Status of Apple blossom thrip (ABT) in apple orchards of Kashmir

\begin{tabular}{|l|l|c|}
\hline S.No & Location & $\begin{array}{c}\text { Range } \\
\text { Incidence/flower }\end{array}$ \\
\hline $\mathbf{1}$ & Madar bandipora & $\mathbf{5 - 8}$ \\
\hline $\mathbf{2}$ & Arin Bandipora & $\mathbf{5 - 7}$ \\
\hline $\mathbf{3}$ & Bagh bandipora & $\mathbf{5 - 6}$ \\
\hline $\mathbf{4}$ & Dangerpora Kullar, anantnag & $\mathbf{3 - 5}$ \\
\hline $\mathbf{5}$ & Luckbown,anantnag & $\mathbf{3 - 6}$ \\
\hline
\end{tabular}


Table.2 Bioefficacy of different pesticides against apple blossom thrip infesting apple variety Red Delicious at Arin in District Bandopora during 2015

\begin{tabular}{|c|c|c|c|c|c|c|c|c|}
\hline \multirow[t]{3}{*}{ Pesticide } & \multirow[t]{3}{*}{$\begin{array}{l}\text { Conc. } \\
(\mathrm{ml} / \mathbf{L})\end{array}$} & \multirow{3}{*}{$\begin{array}{c}\text { Pre } \\
\text { treatment } \\
\text { Count/clu } \\
\text { ster of } \\
\text { flowers }\end{array}$} & \multicolumn{5}{|c|}{$\begin{array}{l}\text { *Mean percent mortality of blossom thrip population over pre } \\
\text { treatment }\end{array}$} & \multirow[t]{3}{*}{$\begin{array}{c}\text { Pooled } \\
\text { mean }\end{array}$} \\
\hline & & & \multicolumn{5}{|c|}{ Days after pesticide treatment } & \\
\hline & & & $1^{\text {st }}$ & $3^{\text {rd }}$ & $5^{\text {th }}$ & $7^{\text {th }}$ & $10^{\text {th }}$ & \\
\hline \multirow{3}{*}{ Thiocloroprid } & 0.3 & 12.33 & $\begin{array}{l}51.11 \\
(45.63)\end{array}$ & $\begin{array}{l}74.33 \\
(59.55)\end{array}$ & $\begin{array}{l}83.33 \\
(65.90)\end{array}$ & $\begin{array}{l}86.10 \\
(68.11)\end{array}$ & $\begin{array}{l}93.66 \\
(75.41)\end{array}$ & 77.70 \\
\hline & 0.4 & 13.00 & $\begin{array}{c}48.26 \\
(44.26)\end{array}$ & $\begin{array}{c}74.37 \\
(59.37)\end{array}$ & $\begin{array}{l}87.11 \\
(68.95)\end{array}$ & $\begin{array}{l}87.11 \\
(68.95)\end{array}$ & $\begin{array}{c}94.95 \\
(77.01)\end{array}$ & 78.38 \\
\hline & 0.5 & 12.66 & $\begin{array}{c}52.62 \\
(46.50)\end{array}$ & $\begin{array}{c}76.46 \\
(59.76)\end{array}$ & $\begin{array}{c}84.49 \\
(66.80)\end{array}$ & $\begin{array}{c}89.74 \\
(71.31)\end{array}$ & $\begin{array}{c}93.66 \\
(75.41)\end{array}$ & 80.19 \\
\hline $\begin{array}{l}\text { Chlorpyriphos } \\
20 \text { EC }\end{array}$ & $0.02 \%$ & 11.66 & $\begin{array}{l}55.55 \\
(48.18)\end{array}$ & $\begin{array}{l}73.88 \\
(59.26)\end{array}$ & $\begin{array}{c}82.12 \\
(64.98)\end{array}$ & $\begin{array}{l}90.87 \\
(72.41)\end{array}$ & $\begin{array}{c}96.29 \\
(78.89)\end{array}$ & 79.74 \\
\hline $\begin{array}{l}\text { Dimethoate } 30 \\
\text { EC }\end{array}$ & $0.05 \%$ & 11.66 & $\begin{array}{c}46.83 \\
(43.18)\end{array}$ & $\begin{array}{c}74.33 \\
(59.55)\end{array}$ & $\begin{array}{l}81.66 \\
(64.64)\end{array}$ & $\begin{array}{l}81.66 \\
(64.64)\end{array}$ & $\begin{array}{c}97.91 \\
(81.62)\end{array}$ & 76.47 \\
\hline Water & check & 11.00 & $\begin{array}{c}3.09 \\
(10.11)\end{array}$ & $\begin{array}{c}3.09 \\
(10.11)\end{array}$ & $\begin{array}{c}6.09 \\
(14.28)\end{array}$ & $\begin{array}{c}3.09 \\
(10.11)\end{array}$ & $\begin{array}{c}9.09 \\
(17.53)\end{array}$ & 4.89 \\
\hline \multicolumn{2}{|c|}{ CD at $5 \%$} & & 1.67 & 2.04 & 1.56 & 1.98 & 2.76 & \\
\hline
\end{tabular}

Table.3 Bioefficacy of different pesticides against apple blossom thrip infesting apple variety Red Delicious at Arin Bandipora during 2016

\begin{tabular}{|c|c|c|c|c|c|c|c|c|}
\hline \multirow[t]{3}{*}{ Pesticide } & \multirow[t]{3}{*}{$\begin{array}{l}\text { Conc. } \\
(\mathrm{ml} / \mathrm{L})\end{array}$} & \multirow{3}{*}{$\begin{array}{c}\text { Pre } \\
\text { treatme } \\
\text { nt } \\
\text { Count/cl } \\
\text { uster of } \\
\text { flowers }\end{array}$} & \multicolumn{5}{|c|}{$\begin{array}{c}\text { *Mean percent mortality of blossom thrip population } \\
\text { over pre treatment }\end{array}$} & \multirow[t]{3}{*}{$\begin{array}{c}\text { Pooled } \\
\text { mean }\end{array}$} \\
\hline & & & \multicolumn{5}{|c|}{ Days after pesticide treatment } & \\
\hline & & & $1^{\text {st }}$ & $3^{\text {rd }}$ & $5^{\text {th }}$ & $7^{\text {th }}$ & $10^{\text {th }}$ & \\
\hline \multirow{3}{*}{$\begin{array}{l}\text { Thiocloropr } \\
\text { id }\end{array}$} & 0.3 & 9.00 & $\begin{array}{c}20.77 \\
(27.10)\end{array}$ & $\begin{array}{c}63.00 \\
(52.52)\end{array}$ & $\begin{array}{c}77.77 \\
(61.86)\end{array}$ & $\begin{array}{c}81.55 \\
(64.45)\end{array}$ & $\begin{array}{c}92.66 \\
(73.95)\end{array}$ & 67.15 \\
\hline & 0.4 & 8.66 & $\begin{array}{c}23.09 \\
(28.71)\end{array}$ & $\begin{array}{c}61.59 \\
(51.59)\end{array}$ & $\begin{array}{c}80.83 \\
(64.02)\end{array}$ & $\begin{array}{c}80.83 \\
(63.92)\end{array}$ & $\begin{array}{c}92.37 \\
(74.27)\end{array}$ & 67.75 \\
\hline & 0.5 & 9.00 & $\begin{array}{c}33.33 \\
(35.25)\end{array}$ & $\begin{array}{c}66.06 \\
(54.35)\end{array}$ & $\begin{array}{c}87.77 \\
(70.86)\end{array}$ & $\begin{array}{c}88.88 \\
(70.40)\end{array}$ & $\begin{array}{c}95.66 \\
(74.27)\end{array}$ & 79.34 \\
\hline $\begin{array}{l}\text { Chlorpyrip } \\
\text { hos 20 EC }\end{array}$ & $0.02 \%$ & 10.66 & $\begin{array}{c}43.71 \\
(41.38)\end{array}$ & $\begin{array}{c}71.85 \\
(57.95)\end{array}$ & $\begin{array}{c}81.23 \\
(64.31)\end{array}$ & $\begin{array}{c}87.52 \\
(69.19)\end{array}$ & $\begin{array}{c}96.90 \\
(79.85)\end{array}$ & 72.85 \\
\hline $\begin{array}{l}\text { Dimethoate } \\
\text { 30 EC }\end{array}$ & $0.05 \%$ & 9.33 & $\begin{array}{c}35.69 \\
(36.68)\end{array}$ & $\begin{array}{c}67.84 \\
(55.44)\end{array}$ & $\begin{array}{c}78.56 \\
(62.41)\end{array}$ & $\begin{array}{c}89.28 \\
(70.76)\end{array}$ & $\begin{array}{c}92.92 \\
(74.53)\end{array}$ & 72.87 \\
\hline Water & check & 9.33 & 0.00 & 0.00 & 0.00 & 0.00 & 0.00 & 0.00 \\
\hline \multicolumn{2}{|c|}{ CD at $5 \%$} & & 2.67 & 2.04 & 1.76 & 1.38 & 2.10 & \\
\hline
\end{tabular}


Table.4 Bioefficacy of different pesticides against apple blossom thrip infesting apple variety Red Delicious at Dangerpora Kullar in District Anantnag during 2015

\begin{tabular}{|c|c|c|c|c|c|c|c|c|}
\hline \multirow[t]{3}{*}{ Pesticide } & \multirow[t]{3}{*}{$\begin{array}{l}\text { Conc. } \\
(\mathrm{ml} / \mathrm{L})\end{array}$} & \multirow{3}{*}{$\begin{array}{c}\text { Pre } \\
\text { treatment } \\
\text { Count/cluster } \\
\text { of flowers }\end{array}$} & \multicolumn{5}{|c|}{$\begin{array}{l}\text { *Mean percent mortality of blossom thrip } \\
\text { population over pre treatment }\end{array}$} & \multirow[t]{3}{*}{$\begin{array}{c}\text { Pooled } \\
\text { mean }\end{array}$} \\
\hline & & & \multicolumn{5}{|c|}{ Days after pesticide treatment } & \\
\hline & & & $1^{\text {st }}$ & $\mathbf{3}^{\text {rd }}$ & $5^{\text {th }}$ & $7^{\text {th }}$ & $10^{\text {th }}$ & \\
\hline \multirow{3}{*}{ Thiocloroprid } & 0.3 & $\begin{array}{c}9.62 \\
(3.09)\end{array}$ & $\begin{array}{c}76.88 \\
(61.27)\end{array}$ & $\begin{array}{c}84.95 \\
(67.29)\end{array}$ & $\begin{array}{c}90.66 \\
(72.29)\end{array}$ & $\begin{array}{c}95.45 \\
(77.76)\end{array}$ & $\begin{array}{c}98.48 \\
(84.98)\end{array}$ & $\begin{array}{c}89.29 \\
(72.72)\end{array}$ \\
\hline & 0.4 & $\begin{array}{l}11.31 \\
(3.35)\end{array}$ & $\begin{array}{c}86.89 \\
(68.83)\end{array}$ & $\begin{array}{l}91.46 \\
(73.11)\end{array}$ & $\begin{array}{c}94.98 \\
(77.09)\end{array}$ & $\begin{array}{c}99.26 \\
(87.53)\end{array}$ & & $\begin{array}{c}94.51 \\
(79.31)\end{array}$ \\
\hline & 0.5 & $\begin{array}{l}11.19 \\
(3.34)\end{array}$ & $\begin{array}{c}93.08 \\
(74.90)\end{array}$ & $\begin{array}{l}95.90 \\
(78.42)\end{array}$ & $\begin{array}{c}99.36 \\
(87.69)\end{array}$ & & $\begin{array}{l}100.00 \\
(90.00)\end{array}$ & $\begin{array}{c}97.67 \\
(84.20)\end{array}$ \\
\hline $\begin{array}{c}\text { Chlorpyriphos } \\
20 \text { EC }\end{array}$ & $0.02 \%$ & $\begin{array}{l}10.37 \\
(3.21)\end{array}$ & $\begin{array}{l}88.68 \\
(70.34)\end{array}$ & $\begin{array}{l}94.12 \\
(76.10)\end{array}$ & $\begin{array}{l}96.73 \\
(79.77)\end{array}$ & $\begin{array}{c}99.54 \\
(88.04)\end{array}$ & $\begin{array}{l}100.00 \\
(90.00)\end{array}$ & $\begin{array}{c}95.74 \\
(80.85)\end{array}$ \\
\hline $\begin{array}{c}\text { Dimethoate } 30 \\
\text { EC }\end{array}$ & $0.05 \%$ & $\begin{array}{l}12.81 \\
(3.57)\end{array}$ & $\begin{array}{c}88.80 \\
(70.47)\end{array}$ & $\begin{array}{c}94.20 \\
(76.21)\end{array}$ & $\begin{array}{c}98.64 \\
(85.29)\end{array}$ & $\begin{array}{l}100.00 \\
(90.00)\end{array}$ & $\begin{array}{l}100.00 \\
(90.00)\end{array}$ & $\begin{array}{c}96.32 \\
(82.39)\end{array}$ \\
\hline Water & check & $\begin{array}{l}10.87 \\
(3.29)\end{array}$ & $\begin{array}{c}38.17 \\
(38.15)\end{array}$ & $\begin{array}{c}28.57 \\
(32.30)\end{array}$ & $\begin{array}{c}23.08 \\
(28.70)\end{array}$ & $\begin{array}{c}21.12 \\
(27.37)\end{array}$ & $\begin{array}{c}18.51 \\
(25.43)\end{array}$ & $\begin{array}{c}25.89 \\
(30.39)\end{array}$ \\
\hline \multicolumn{2}{|c|}{ CD at $5 \%$} & $\begin{array}{c}2.67 \\
(0.40)\end{array}$ & $\begin{array}{c}3.47 \\
(2.79)\end{array}$ & $\begin{array}{c}3.30 \\
(3.20)\end{array}$ & $\begin{array}{c}2.52 \\
(5.13)\end{array}$ & $\begin{array}{c}1.72 \\
(4.31)\end{array}$ & $\begin{array}{c}2.08 \\
(3.81)\end{array}$ & \\
\hline
\end{tabular}

Table.5 Bioefficacy of different pesticides against apple blossom thrip infesting apple variety Red Delicious at Luckbown in District Anantnag during 2017

\begin{tabular}{|c|c|c|c|c|c|c|c|c|}
\hline \multirow[t]{3}{*}{ Pesticide } & \multirow[t]{3}{*}{$\begin{array}{l}\text { Conc. } \\
(\mathrm{ml} / \mathrm{L})\end{array}$} & \multirow{3}{*}{$\begin{array}{c}\text { Pre } \\
\text { treatment } \\
\text { Count/cluster } \\
\text { of flowers }\end{array}$} & \multicolumn{5}{|c|}{$\begin{array}{l}\text { *Mean percent mortality of blossom thrip } \\
\text { population over pre treatment }\end{array}$} & \multirow[t]{3}{*}{$\begin{array}{c}\text { Pooled } \\
\text { mean }\end{array}$} \\
\hline & & & \multicolumn{5}{|c|}{ Days after pesticide treatment } & \\
\hline & & & $1^{\text {st }}$ & $3^{\text {rd }}$ & $5^{\text {th }}$ & $7^{\text {th }}$ & $10^{\text {th }}$ & \\
\hline \multirow{3}{*}{ Thiocloroprid } & 0.3 & $\begin{array}{c}9.87 \\
(3.14)\end{array}$ & $\begin{array}{l}79.16 \\
(62.85)\end{array}$ & $\begin{array}{c}85.64 \\
(67.80)\end{array}$ & & $\begin{array}{c}94.35 \\
(76.26)\end{array}$ & & \\
\hline & 0.4 & $\begin{array}{l}10.00 \\
(3.16)\end{array}$ & $\begin{array}{l}86.08 \\
(68.19)\end{array}$ & $\begin{array}{c}90.62 \\
(78.56)\end{array}$ & $\begin{array}{c}96.26 \\
(85.42)\end{array}$ & & & $\begin{array}{c}94.34 \\
(82.43)\end{array}$ \\
\hline & 0.5 & $\begin{array}{l}10.37 \\
(3.22)\end{array}$ & $\begin{array}{c}91.17 \\
(72.91)\end{array}$ & $\begin{array}{c}95.93 \\
(78.56)\end{array}$ & $\begin{array}{l}98.90 \\
(85.74)\end{array}$ & & & \\
\hline $\begin{array}{l}\text { Chlorpyriphos } \\
20 \text { EC }\end{array}$ & $0.02 \%$ & $\begin{array}{l}10.25 \\
(3.20)\end{array}$ & $\begin{array}{c}89.63 \\
(71.23)\end{array}$ & $\begin{array}{c}93.94 \\
(75.79)\end{array}$ & $\begin{array}{c}98.27 \\
(84.70)\end{array}$ & $\begin{array}{c}99.43 \\
(87.83)\end{array}$ & $\begin{array}{l}100.00 \\
(90.00)\end{array}$ & $\begin{array}{c}96.25 \\
(81.91)\end{array}$ \\
\hline $\begin{array}{c}\text { Dimethoate } 30 \\
\text { EC }\end{array}$ & $0.05 \%$ & $\begin{array}{l}9.75 \\
(3.12)\end{array}$ & $\begin{array}{c}86.65 \\
(68.66)\end{array}$ & $\begin{array}{c}92.43 \\
(74.18)\end{array}$ & $\begin{array}{c}96.93 \\
(81.32)\end{array}$ & $\begin{array}{c}99.38 \\
(87.73)\end{array}$ & $\begin{array}{l}100.00 \\
(90.00)\end{array}$ & $\begin{array}{c}95.08 \\
(80.38)\end{array}$ \\
\hline Water & check & $\begin{array}{c}9.44 \\
(3.07)\end{array}$ & $\begin{array}{c}29.30 \\
(32.76)\end{array}$ & $\begin{array}{c}25.84 \\
(30.53)\end{array}$ & $\begin{array}{c}21.99 \\
(27.94)\end{array}$ & $\begin{array}{c}19.19 \\
(25.96)\end{array}$ & $\begin{array}{c}15.72 \\
(23.25)\end{array}$ & $\begin{array}{c}22.41 \\
(28.09)\end{array}$ \\
\hline \multicolumn{2}{|c|}{ CD at $5 \%$} & 1.39 & 3.33 & 3.90 & 2.94 & 1.78 & 2.52 & \\
\hline
\end{tabular}


Table.6 Cumulative bio-efficacy of pesticides against apple blossom thrip infesting apple variety Red Delicious in both the Districts (Anantnag and Bandipora) during 2015 \& 2017 Both Locations

\begin{tabular}{|l|c|c|c|c|}
\hline \multirow{2}{*}{ Pesticide } & \multirow{2}{*}{$\begin{array}{c}\text { Conc. } \\
(\mathrm{ml} / \mathrm{L})\end{array}$} & \multicolumn{3}{|c|}{$\begin{array}{c}\text { Mean percent mortality of } \\
\text { (Apple blossom thrip) }\end{array}$} \\
\cline { 3 - 5 } & & $\begin{array}{c}\text { Anantnag } \\
(\mathbf{2 0 1 5}-16)\end{array}$ & $\begin{array}{c}\text { Bandipora } \\
(\mathbf{2 0 1 5} \text { \&17) }\end{array}$ & Mean \\
\hline \multirow{2}{*}{ Thiocloroprid } & 0.3 & $\mathbf{8 9 . 4 8}$ & $\mathbf{7 2 . 4 2}$ & $\mathbf{8 0 . 9 5}$ \\
\cline { 2 - 5 } & 0.4 & $\mathbf{9 4 . 4 2}$ & $\mathbf{7 3 . 0 6}$ & $\mathbf{8 3 . 7 4}$ \\
\hline Chlorpyriphos 20 & 0.5 & $\mathbf{9 7 . 4 3}$ & $\mathbf{7 9 . 7 6}$ & $\mathbf{8 6 . 5 9}$ \\
\hline EC & $0.02 \%$ & $\mathbf{9 5 . 9 9}$ & & $\mathbf{7 6 . 2 9}$ \\
\hline Dimethoate 30 EC & $0.05 \%$ & $\mathbf{9 5 . 7 0}$ & $\mathbf{7 4 . 6 7}$ & $\mathbf{1 3 . 7 9}$ \\
\hline Water & check & $\mathbf{2 4 . 1 5}$ & $\mathbf{3 . 4 4}$ & \\
\hline
\end{tabular}

Fig.1

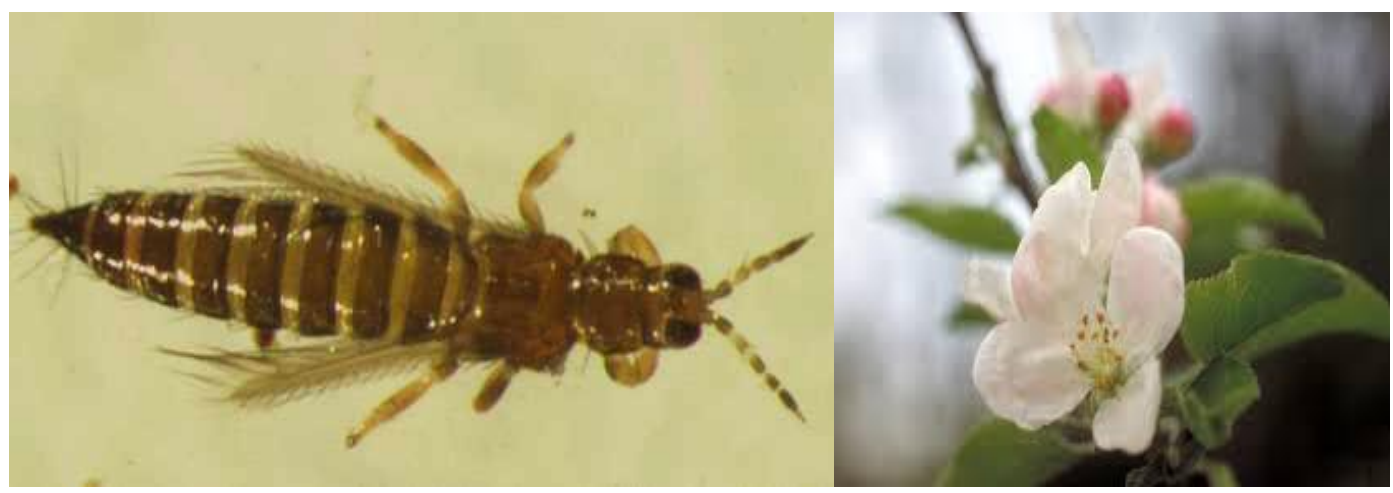

Fig.2 Effect of different insecticides on yield of apple variety Red Delicious in District (Bandipora and Anantnag) 2015 \& 2017

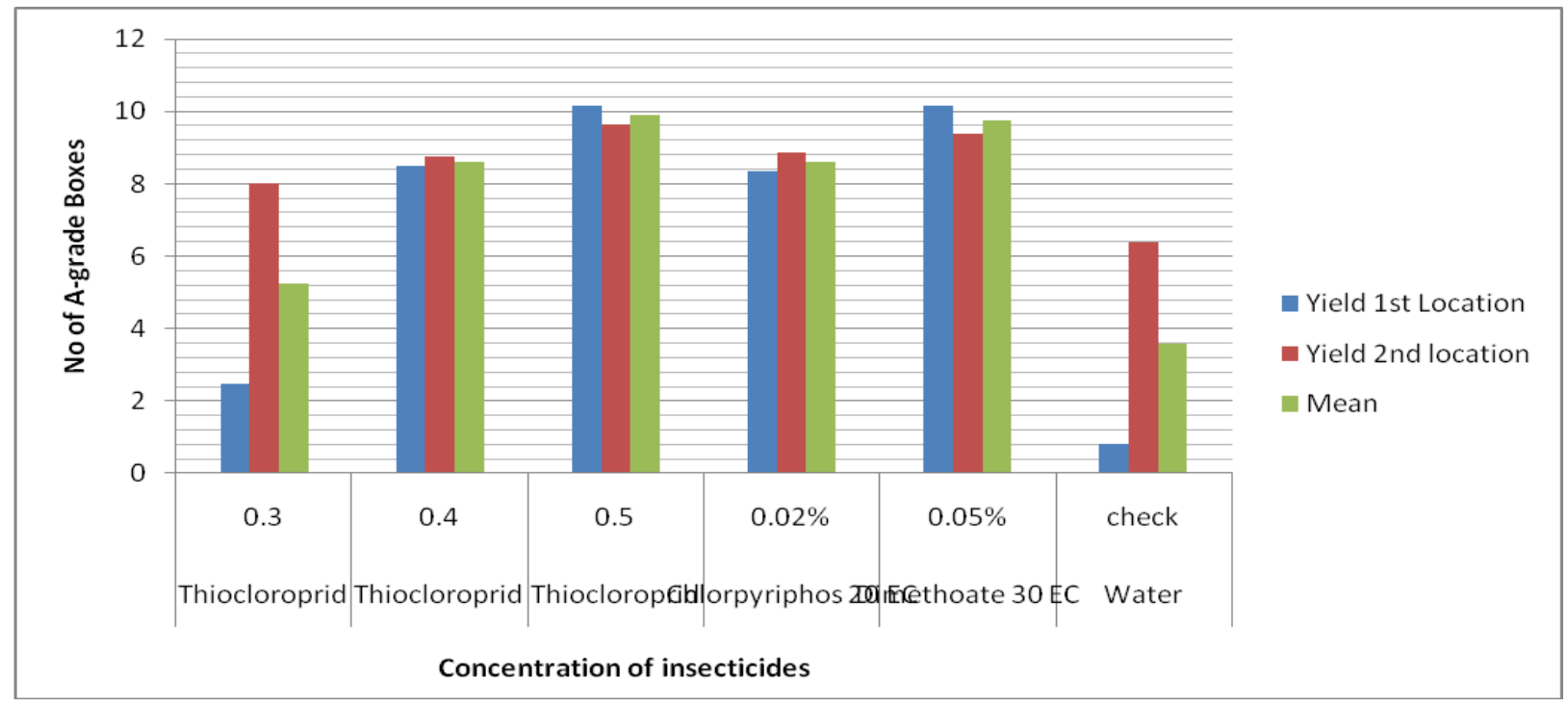


Pooled data on bio-efficacy of Thiocloroprid against apple blossom thrip on apple during 2015-2017 (Bandipora and Anantnag)

When the data of two districts (Bandipora and Anantnag) was pooled together, it was found that thiocloroprid, chlorpyriphos $20 \mathrm{EC}$ and dimethoate 30 EC resulted 80.95, 83.74, $88.59,86.14$ and $85.18 \%$ mortality of the apple blossom thrip (ABT) population at 0.3 , 0.4 and $0.5 \mathrm{ml} / \mathrm{L}$ of water; 0.02 and $0.05 \%$ concentrations in comparison to check $(13.79 \%)$ in District Bandipora, respectively (Table 6).

In conclusion, the pest is serious threat to apple growing belts in Bandipora and some areas of Anantnag near the foot hill regions. The control strategy was developed by selecting some pesticides above. It was found that all insecticides are giving best control. Similar findings by Singh (1989) revealed by using six insecticides against Thrips carthami and thrips flavus on apple using one application at the green tip stage. The Thiocloroprid were found best in terms of mortality achieved.

\section{References}

Bhalla O P and Thakur J R. 1974. Note on thrips fauna in Himachal Pradesh. Himachal Journal of Agricultural Research 2(2): 2-4.

Fennah, R G., 1965, The influence of environmental stress on the cacao tree in predetermining the feeding sites of cacao thrips, Selenothrips rubrocinctus (Giard) on leaves and pods. Bull. Ent. Res., 56: 333349.

Fernando, H.E. and Peiris, J.W.L., 1957, Investigations on the chilli leaf curl complex and its control. Trop. Agric., 113 (4): 305-325.

Flowers, RW. 1989, The occurrence of the citrus thrips, Scirtothrips citri (Thysanoptera: Thripidae) in Florida. Florida Entomologist,
72(2): 385 .

Gal, D., Kachlon, Y., Hadar, A., Rosenfeld, G., Cohen, Y. and Balchinsky, D., 2000, Damage in table grapes caused by western flower thrips (Frankliniella occidentalis) prior to vintage. Alon. Hanotea., 54(5): 181-183.

Gilstrap, F.E. and Oaten, E.R, 1976, The bionomics of Scolothrips sexmaculatus (Pergande) (Thysanoptera: Thripidae), an insect predator of spider mites. Hilgardia, 44: 27-59.

Gonzalez, R.H., 1996, A new pest of stone fruits and table grapes in Chile: Frankliniella occidentalis (Pergande) (Thysanoptera: Thripidae). Revista. Fruticola, 16(3): 107111.

Grassell Y, D., 1996, The thrips Frankliniella occidentalis in vegetable and fruit crops. Phytoma, 482: 42-43.

Hall, W.J., 1930, The South African Citrus thrips in Southern Rhodesia. Rep: Mazoe Citrus Exp. Stn., 1: 5-55.

Harish, R., 2002, Species complex, biology and management of thrips on grapes $\mathrm{cv}$. Bangalore blue, M. Sc. (Agri.) Thesis, univ, Agric, sci., Bangalore.

Harris, H.M., Drake, C.J. and Tate, H.D., 1936, Observation on the onion thrips. Iowa College Journal of Science, 10: 155-172.

Hashimoto, S., Mizosimha, S. and Kohno, M., 1984, Studies on the ecology and the control of yellow tea thrips Scirtothrips dorsalis, 2. Damage and control of the thrips. Proceedings Association for plant protection of Kyushu, 30: 164-166.

Immaraju, J.A. and Morse, J.G., 1990, Selection for pyrethroid resistance, reversion and cross-resistance with citrus thrips (Thysanoptera: Thripidae). J. Econ. Ent., 83: 698-704.

Jaganmohan, N., Krishnaiah, K. and Prasad, V.G., 1980, Evaluation of insecticides for the control of pests of sweet pepper (Capsicum annuum Var. grossum Linn.). Pestology, 4: 13-18.

Jarande, N.T. and Dethe, M.D., 1994, Imidacloprid for effective control of sucking pests of chilli. Pestology, 18: 1517. 
Kandasamy, C., Mohanasundaram, M. and Karuppuchamy, P., 1990, Evaluation of insecticides for the control of thrips Scirtothrips dorsalis Hood on chillies (Capsicum annuum L). Madras Agric. J., 77 (3\&4): 169-172.

Keisa, T.J. and Varatharajan, R., 1995, Efficacy of two neem products in the field control of Scirtothrips dorsalis Hood (Thysanoptera) on Capsicum annuum L. Indian J. Plant Prot., 23: 166-168.

Khaire, V.A. and Naik, R.L., 1986, Comparative efficacy of certain newer pesticides against the sucking pest complex on chilli. South Indian Hort., 33(6): 402-403.

Khaire, V.A., Pokharkar, R.N. and Lawander, K.E., 1989, Studies on the efficacy of certain insecticides against thrips (Scirtothrips dorsalis Hood) on capsicum (Capsicum annum). Pestology, 13 (7): 2223.

Kirk, W.D.J., 1985b, Aggregation and mating of thrips in flowers of Calystega sepium. Econ. Entomol., 10: 433-440.

Kirk, W.D.J., 1990, Body size in flower thrips. In: Proceedings of the third International Symposium on Thysanoptera, Kazimierz, Dolny, Poland. Warsaw Agricultural University Press, Warsaw, pp. 65-69.

Kulakarni, N.S. and Adsule, P.G., 2006, Evaluation of Polo 50SC (diafenthiuron) on the management of thrips and mites in grapes. Pestology, 30 (5):30-32.

Morishita, M. 2001. Toxicity of some insecticides to larvae of Flankliniella occidentalis (Pergande) (Thysanoptera: Thripidae) evaluated by the Petri dish-spraying tower method. Appl Entomol Zool 36: 137-141.

Moritz, G. 1997. Structure, growth and development, pp. 15-64. In T. Lewis [ed.],
Thrips as crop pests. CAB International, Wallingford, UK. Moritz, G., D. C. Morris, and L. A. Mound. 2001. Thrips ID Pest of the world. An interactive identification and information system. CSIRO Publishing. Collingwood, Victoria, Australia.

Moritz, G., L. A. Mound, D. C. Morris, and A. Goldarazena. 2004b. Pest thrips of the world. Visual and Molecular identification of pests thrips. An identification and information system using molecular and microscopical methods. Lucid- CSIRO publishing, Collingwood, Victoria, Australia.

Moritz, G., S. Kumm, and L. Mound. 2004a. Tospovirus transmission depends on thrips ontogeny. Virus Research 100: 143-149.

Morse, J. G., and O. L. Brawner. 1986. Toxicity of pesticides to Scirtothrips citri (Thysanoptera: Thripidae) and implications to resistance management. J Econ Entomol 79: 565-570.

Mound, L. A. 1997. Biological diversity, pp. 197216. In T. Lewis [ed.], Thrips as crop pests. CAB International, Wallingford, UK. Mound, L. A. 2005. Thysanoptera: Diversity and interactions. Annu Rev Entomol 50: 247-269.

Singh Mahabir, Singh M 1989. Chemical control of blossom thrips in apple. Indian Journal of Entomology 49: 580-582.

Verma S K., Phogat K P S. 1994 Evaluation of some insecticides for the control of apple blossom thrips (Thrips spp.) Progressive Horticulture 26: 98-103.

Verma, K. L 1979 Apple blossom thrips and their control. Pesticides. 13(1): 32-33.

\section{How to cite this article:}

Muneer Ahmad, M. Ayoub Manto, Sajad Mohu Din and Pathania, S.S. 2019. New Approaches of Management for Apple Blossom Thrip (ABT) in Apple Orchards of Kashmir Valley. Int.J.Curr.Microbiol.App.Sci. 8(01): 1573-1580. doi: https://doi.org/10.20546/ijcmas.2019.801.165 